\title{
Wideband, Low-Profile, Dual-Polarized Slot Antenna with an AMC Surface for Wireless Communications
}

\author{
Wei Hu, ${ }^{1,2}$ Rui-Na Lian, ${ }^{1}$ Zhao-Yang Tang, ${ }^{1}$ and Ying-Zeng Yin' \\ ${ }^{1}$ National Laboratory of Science and Technology on Antennas and Microwaves, Xidian University, Xian, Shaanxi 710071, China \\ ${ }^{2}$ Collaborative Innovation Center of Information Sensing and Understanding, Xidian University, Xian, Shaanxi 710071, China
}

Correspondence should be addressed to Wei Hu; mwhuwei@163.com

Received 22 July 2016; Revised 27 September 2016; Accepted 4 October 2016

Academic Editor: Renato Cicchetti

Copyright (C) 2016 Wei Hu et al. This is an open access article distributed under the Creative Commons Attribution License, which permits unrestricted use, distribution, and reproduction in any medium, provided the original work is properly cited.

A wideband dual-polarized slot antenna loaded with artificial magnetic conductor (AMC) is proposed for WLAN/WIMAX and LTE applications. The slot antenna mainly consists of two pairs of arrow-shaped slots along the diagonals of the square patch. Stepped microstrip feedlines are placed orthogonally to excite the horizontal and vertical polarizations of the antenna. To realize unidirectional radiation and low profile, an AMC surface composed of $7 \times 7$ unit cells is designed underneath a distance of $0.09 \lambda_{0}$ ( $\lambda_{0}$ being the wavelength in free space at $2.25 \mathrm{GHz}$ ) from the slot antenna. Both the dual-polarized slot antenna and the AMC surface are fabricated and measured. Experimental results demonstrate that the proposed antenna achieves for both polarizations a wide impedance bandwidth (return loss $10 \mathrm{~dB}$ ) of $36.7 \%$, operating from 1.96 to $2.84 \mathrm{GHz}$. The isolation between the two input ports keeps higher than $29 \mathrm{~dB}$ whereas the cross-polarization levels basically maintain lower than $-30 \mathrm{~dB}$ across the entire frequency band. High front-to-back ratios better than $22 \mathrm{~dB}$ and a stable gain higher than $8 \mathrm{dBi}$ are obtained over the whole band.

\section{Introduction}

Dual-polarized antennas have led to a wide range of applications in modern wireless communication systems since they can effectively mitigate multipath fading problem and enhance channel capacity. To cover several wireless communication services, the antenna should be designed to operate in a wide band. Furthermore, the space to accommodate antennas becomes more and more limited, resulting in high demand for low-profile antennas. Nevertheless, it is still difficult to design a low-profile antenna to simultaneously achieve wide band, high port isolation, and low crosspolarization. Microstrip antenna is a good candidate for base-station application due to its light weight, low cost, low profile, and easy conformability for dual-polarization implementation [1-3]. As known, both the bandwidth and port isolation of the microstrip antenna are not good enough. In recent years, various attractive feeding mechanisms, such as meandering probes [4], L-probes [5], T-type probes [6], $\Gamma$-shaped probes [7], hybrid feeding mechanisms [8, 9], aperture-coupled feeds [10], and differential feeds [11, 12], are proposed to enhance the performance of dual-polarized patch antennas. However, all these techniques lead to high profiles and nonplanar feeding structures, which bring great difficulties in integration and fabrication. In [13], a wideband dual-polarized patch antenna employing a broadband balun has been proposed. Unfortunately, it is hard to design a balun suitable to cover all the requirements of wideband dualpolarized antennas.

By contrast, slot antenna has a planar structure but with high back radiation [14]. For unidirectional radiation, a metallic ground plane is needed to suppress back radiation. However, when the antenna is located close to the metallic plane its radiation efficiency reduces because of the electromagnetic energy reflection due to the metal reflector. To tackle the problem, the antenna is generally placed at a distance of $0.25 \lambda$ or higher from the metallic ground plane, and this results in a high profile which may be impractical for modern wireless communication systems. Recently, the artificial magnetic conductor (AMC) surface attracts much interest owing to its good properties including in-phase reflection for plane waves and suppression of surface waves. AMC can act as a reflector for enhanced radiations in the frequency range where it reflects the incoming 


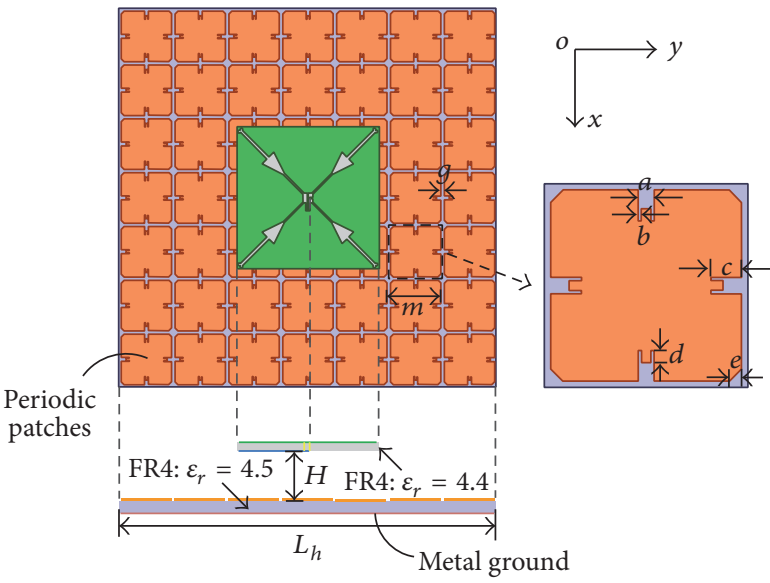

(a)

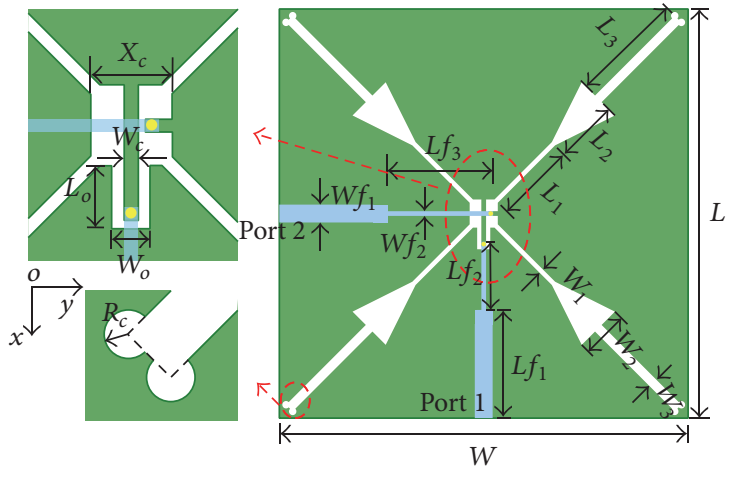

Top layer

Bottom layer

Via

(b)

FIgURE 1: Geometry of the proposed dual-polarized antenna. (a) Top and side views and (b) dual-polarized slot antenna.

radiations with a phase coefficient ranging between $-90^{\circ}$ and $+90^{\circ}[15,16]$. Additionally, the antenna works well when it is positioned very closely to the AMC surface, which makes it possible to design an antenna having a low profile.

In our previous study [17], a stepped-impedance slot structure has been successfully employed in the dualpolarized antenna design as a kind of slot resonator to realize broadband operation. In this paper, two pairs of arrowshaped slots are instead introduced along the diagonals of a squared patch and combined with an AMC surface, so as to realize a lower profile and a wider impendence bandwidth with respect to the antenna proposed in [17], as well as good dual-polarized operation performance. The paper extends the previous study and provides a new effective approach to the design of wideband low-profile dual-polarized antennas based on radiating slots and AMC surfaces. Furthermore, measured results performed on a prototype show that the antenna exhibits low cross-polarization levels and high port isolation.

\section{Antenna Configuration and Design}

Figure 1 shows the geometry of the proposed antenna. It consists of a dual-polarized slot antenna and an AMC surface located underneath the antenna to act as a reflector. The slot antenna is constructed on a $1 \mathrm{~mm}$ thick FR4 substrate $\left(\varepsilon_{r}=\right.$ $4.4, \tan \delta=0.02$ ) while the AMC structure is printed on a $3 \mathrm{~mm}$ thick FR4 substrate $\left(\varepsilon_{r}=4.5, \tan \delta=0.02\right)$. As shown in Figure 1(b), the slot antenna selects two identical pairs of arrow-shaped slots along the diagonals as the radiation aperture and two orthogonal stepped strips as feedlines. Each arrow-shaped slot, formed by three slotline resonators, is composed of a narrow rectangular slot, a triangular slot, and a slightly wide rectangular slot loaded with two circular slots at its end. It is noteworthy that two-circular-slot design is employed in each arrow-shaped slot to increase the operating bandwidth and improve the impedance matching.
The resonant condition of the antenna depends upon the length $\left(L_{1}+L_{2}+L_{3}\right)$ of the arrow-shaped slot, which is approximately equal to half of the wavelength. Both the length and width of each section of the arrow-shaped slot can be adjusted for the slot antenna to achieve broadband property. In addition, the microstrip feeding lines are provided with stepped strips for a better impedance matching. The excitation network consists of two narrow rectangular strips extending to the end of the stepped feedlines where they electrically join with each other by two slender pins (see Figure 1).

The AMC surface consists of metallic ground, grounded dielectric substrate, and periodical patches, without any grounded via. Generally, the periodicity of the patches is much smaller than the wavelength. The unit cell of the proposed AMC surface, shown in Figure 1(a), has a size of $16.5 \mathrm{~mm} \times 16.5 \mathrm{~mm}$ and exhibits a gap width of $1.5 \mathrm{~mm}$ between adjacent cells. According to the working mechanism of the AMC surface, when the phase of the reflected wave on the AMC surface in the desired band varies from $-90^{\circ}$ to $90^{\circ}$, it presents in-phase property. The performance of an infinite repetition of the unit cell is simulated by ANSYS HFSS. As shown in Figure 2(a), the HFSS model is based on simulating scattering parameters of a single port air-filled waveguide with two perfect electric conductors (PEC) and two perfect magnetic conductors (PMC) walls. The propagating plane wave is polarized parallel to the PMC walls and normal to the PEC walls. Figure 2(b) plots the simulated phase reflection response of the AMC structure for a normally incident plane wave. It can be obviously seen that the $\pm 90^{\circ}$ reflection phase of the AMC structure covers from $1.85 \mathrm{GHz}$ to $3.16 \mathrm{GHz}$. Based upon the unit cell, a $7 \times 7$ periodic AMC array is developed and positioned underneath at a distance of $12 \mathrm{~mm}\left(0.09 \lambda_{0}, \lambda_{0}\right.$ being the wavelength in free space at $2.25 \mathrm{GHz}$ ) from the antenna. This array acts as a reflector to achieve unidirectional radiation patterns with high front-toback ratios. 


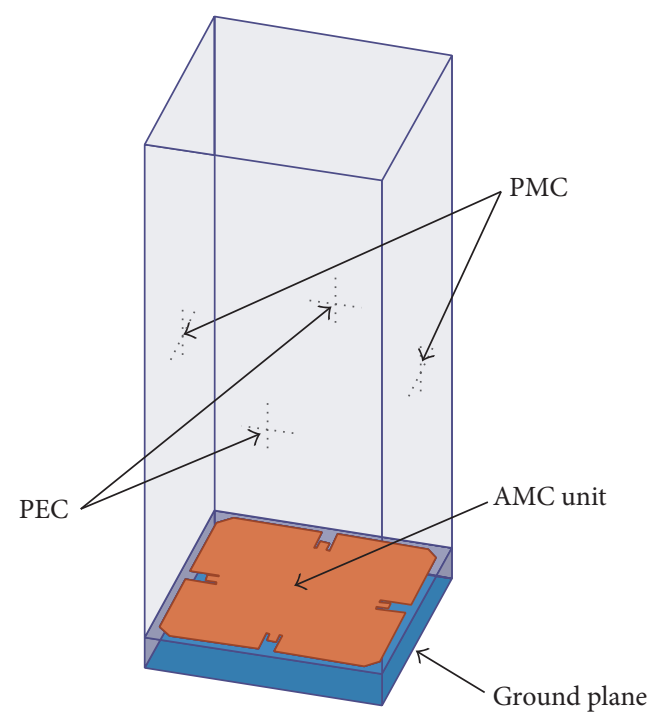

(a)

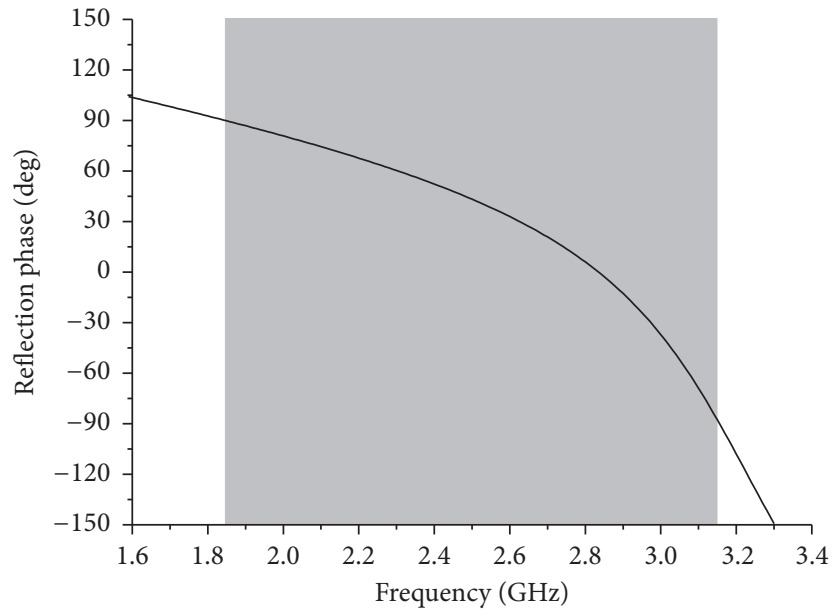

(b)

FIGURE 2: (a) Simulation model of AMC unit. (b) Simulated reflection phase of the proposed AMC unit.

\section{Results and Discussions}

The antenna combined with the AMC structure is analyzed and optimized. The final values of the parameters defined in Figure 1 are listed in Table 1.

To have an insight into the mechanism governing the behavior of the proposed antenna, a detailed study on the antenna without reflector and that over a finite PEC $(30 \mathrm{~mm}$ away with the same size as the AMC reflector) has been performed for comparison. Figure 3 plots the simulated reflection coefficients $\left(\left|S_{11}\right|\right)$ of three dual-polarized slot antennas. As it can be observed, the antenna over the AMC reflector achieves the widest operating bandwidth with good impedance matching. This behavior is confirmed by the input impedance curves exhibited by the different dualpolarized slot antennas depicted in Figure 4. In particular, the impedance curve related with the AMC reflector shows that one extra resonance, with resistance of about $50 \mathrm{Ohm}$ and null reactance at $2.07 \mathrm{GHz}$, is generated by the introduction of the AMC surface, which results in antenna miniaturization and broadband property.

The effects of key design parameters such as $W_{1}, W_{3}$, $H, m$ and the number of AMC units on the antenna $S$ parameter are investigated. In particular, when the narrow rectangular slot width $W_{1}$ increases, the second resonant frequency shifts to higher frequency whereas the first resonant frequency almost keeps unchanged as it appears in Figure 5. Simultaneously, the middle band is significantly deteriorated. This means that $W_{1}$ plays an important role in controlling the second resonant frequency and in the improvement of the impedance matching. Figure 6 shows the frequency behavior of $\left|S_{11}\right|$ of the proposed antenna for different values of parameter $W_{3}$. From this figure it appears that as $W_{3}$ increases from 0.5 to $1.5 \mathrm{~mm}$ the first deep in the resonant frequency shifts up, while the second deep in the resonant frequency increases. Finally, $W_{3}=1 \mathrm{~mm}$ is
TABLE 1: Optimized parameters of the proposed antenna.

\begin{tabular}{lc}
\hline Para. & Values $(\mathrm{mm})$ \\
\hline$L$ & 43.5 \\
$W$ & 43.5 \\
$L_{1}$ & 8.6 \\
$L_{2}$ & 6.8 \\
$L_{3}$ & 12.0 \\
$W_{1}$ & 0.7 \\
$W_{2}$ & 5.0 \\
$W_{3}$ & 1.0 \\
$L f_{1}$ & 12.5 \\
$L f_{2}$ & 6.25 \\
$L f_{3}$ & 10.25 \\
$W f_{1}$ & 1.5 \\
$W f_{2}$ & 0.5 \\
$X_{c}$ & 3.0 \\
$W_{c}$ & 0.5 \\
$L_{o}$ & 2.3 \\
$W_{o}$ & 1.4 \\
$g$ & 1.5 \\
$m$ & 16.5 \\
$H$ & 12.0 \\
$L_{h}$ & 115.5 \\
$a$ & 1.3 \\
$b$ & 0.25 \\
$c$ & 2.5 \\
$d$ & 1.0 \\
$e$ & 1.0 \\
\hline
\end{tabular}

chosen as the optimized value. In the process of designing the antenna with the AMC reflector, the distance $H$ between 


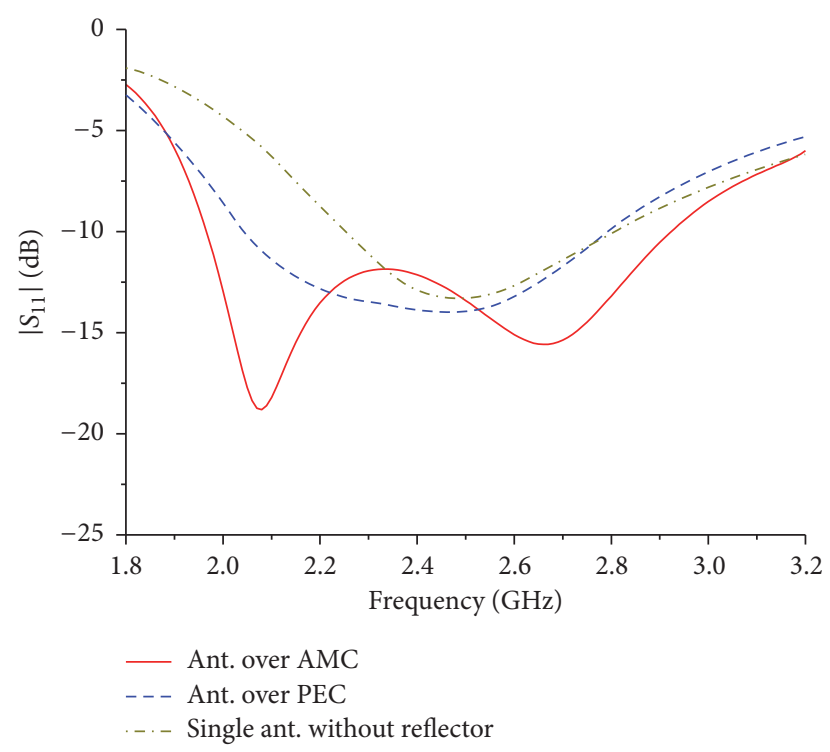

FIGURE 3: Simulated $\left|S_{11}\right|$ of the different dual-polarized slot antennas.

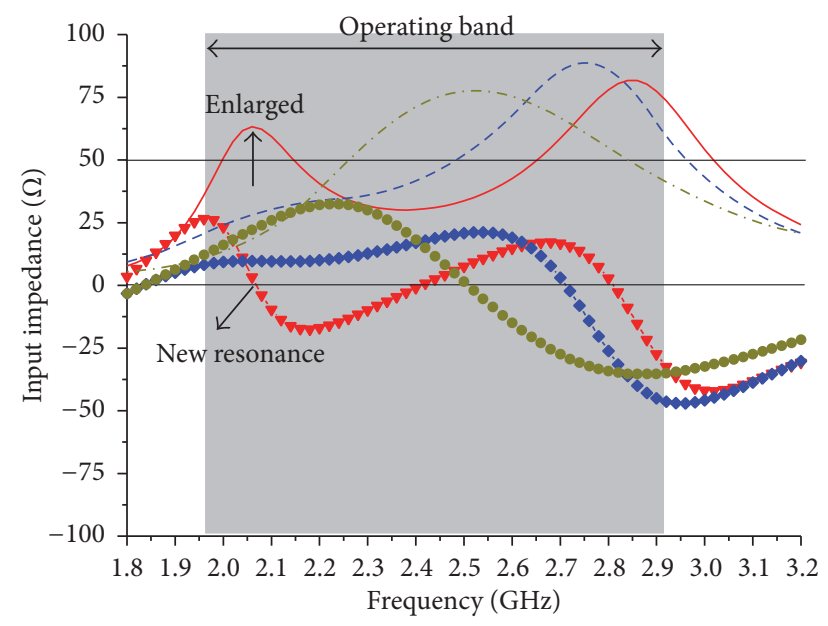

$$
\begin{array}{ll}
\text { Ant. over AMC: } & \text { Single ant.: } \\
\hline \text { - Real } & -- \text { - Real } \\
\rightarrow \text { Imaginary } & \rightarrow-\text { Imaginary } \\
\text { Ant. over PEC: } & \\
-- \text { - Real } & \\
\rightarrow-\text { Imaginary } &
\end{array}
$$

FIGURE 4: Simulated input impedance of the different dual-polarized slot antennas.

the slot antenna and the AMC structure has to be considered. Figure 7 illustrates the frequency behavior of $\left|S_{11}\right|$ of the dual-polarized slot antenna, placed over the AMC plane, for different values of the distance $H$. From this figure it appears that the bandwidth is almost unaffected, while the impedance matching strongly depends on parameter $H$. In particular, an increase of the distance between the antenna and the AMC surface leads to a good impedance matching. For a lowprofile dual-polarized antenna, $H=12 \mathrm{~mm}$ is finally selected. In addition, the frequency behavior of $\left|S_{11}\right|$ of the proposed

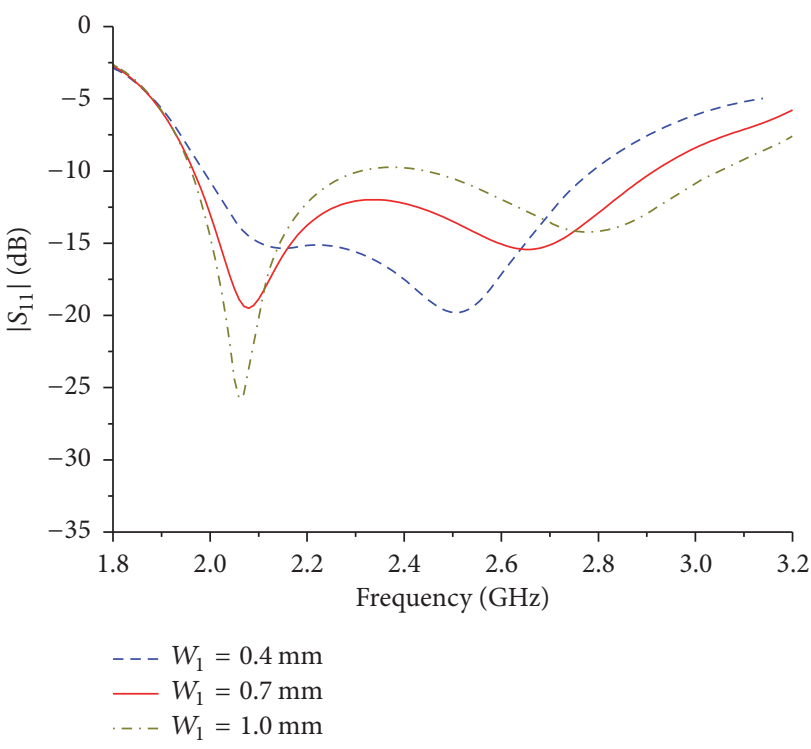

FIGURE 5: Simulated $\left|S_{11}\right|$ of the proposed dual-polarized antenna with different $W_{1}$.

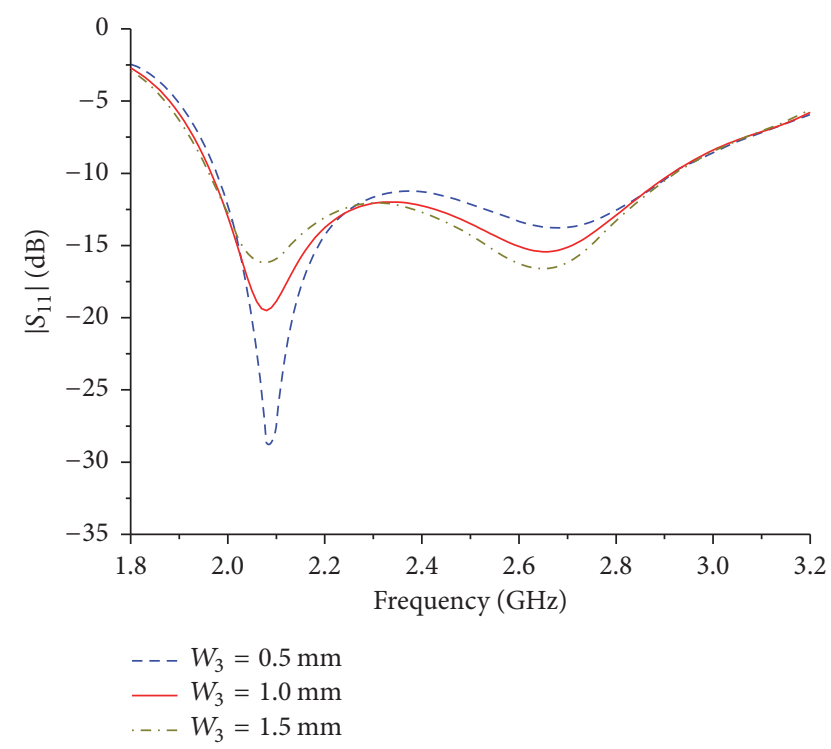

FIGURE 6: Simulated $\left|S_{11}\right|$ of the proposed dual-polarized antenna with different $W_{3}$.

antenna for different sizes of the AMC unit is represented in Figure 8. It is clear that as parameter $m$ increases from 13.5 to $19.5 \mathrm{~mm}$, the operating band of the antenna shifts to lower frequency. When $m$ increase to $19.5 \mathrm{~mm}$, the impedance matching is improved in the lower band but the working bandwidth shrinks obviously at the higher frequency. Thus, the optimum parameter $m$ is set at $16.5 \mathrm{~mm}$. Figure 9 depicts the simulated $\left|S_{11}\right|$ of the proposed antenna with different number of AMC units. From this figure it appears that the number of AMC units mainly affects both the working bandwidth at the higher frequency and impedance matching in the lower band. By balancing the overall dimension of the 


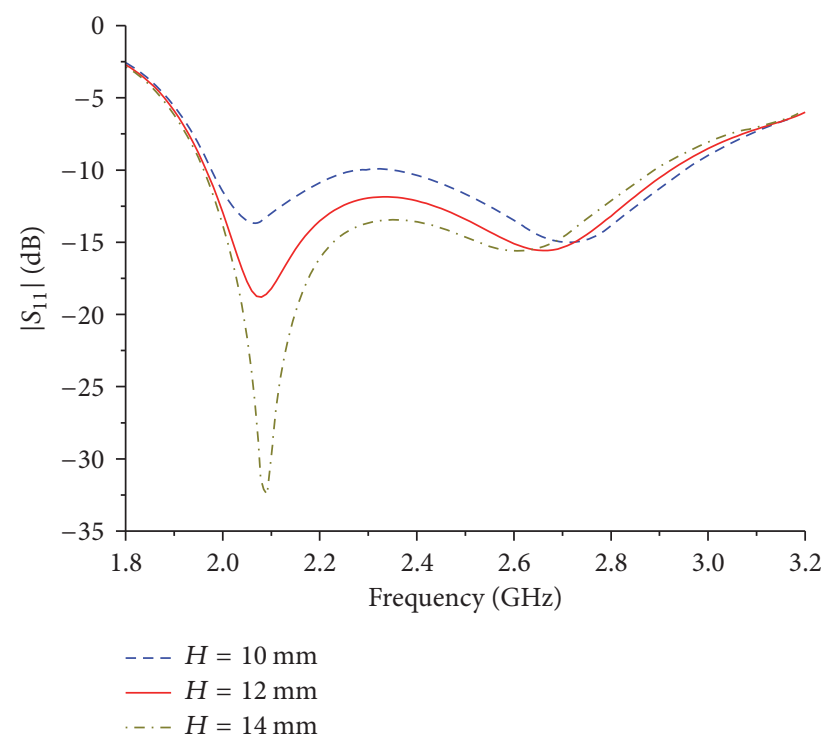

Figure 7: Simulated $\left|S_{11}\right|$ of the proposed dual-polarized antenna with different $H$.



FIGURE 8: Simulated $\left|S_{11}\right|$ of the proposed dual-polarized antenna with different $m$.

AMC surface and the impedance bandwidth of the antenna, a $7 \times 7 \mathrm{AMC}$ array has been finally selected.

To further investigate the dual-polarized operation mechanism of the slot antenna, inspired by [18], the simulated electric-field distributions on the proposed antenna excited by Port 1 and Port 2 at $2.4 \mathrm{GHz}$ are illustrated in Figure 10 . From this figure it appears that the maximum electric-field intensity is concentrated in the two pairs of arrow-shaped slots, meaning that these give the main contributions to the total field radiated by the antenna. So, when Port 1 is excited the resulting electric field is linearly polarized along the $x$ axis (see in Figure 10(a)). Similarly, as shown in Figure 10(b),

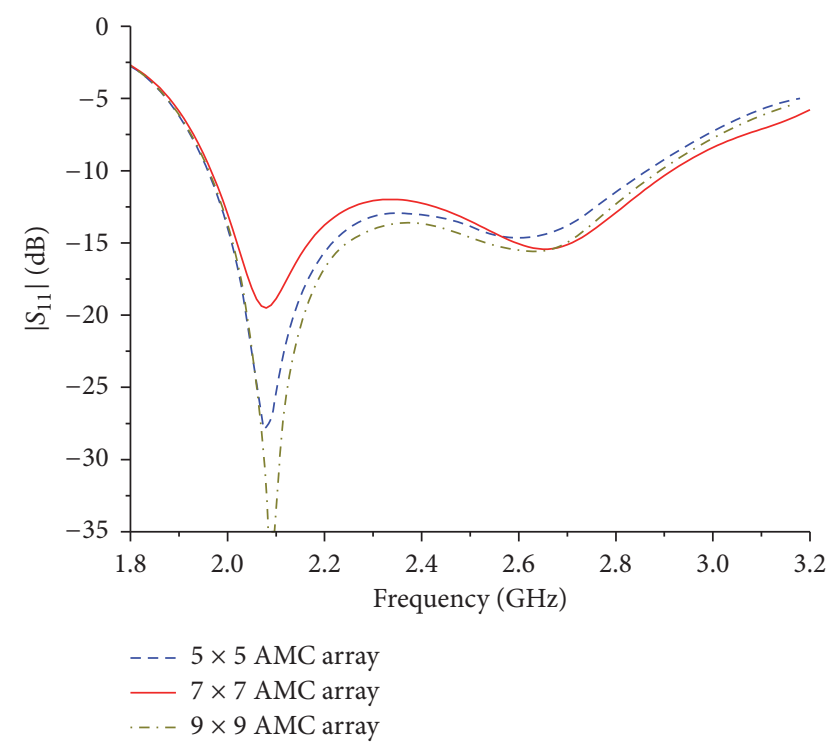

FIGURE 9: Simulated $\left|S_{11}\right|$ of the proposed dual-polarized antenna with different number of AMC units.

the $y$-directed linearly polarized radiation is produced when Port 2 is excited.

To validate the design concept, the proposed dualpolarized slot antenna employing an AMC structure as reflector has been realized and measured. The photograph of the antenna prototype is depicted in Figure 11. In Figure 12 the frequency behavior of the computed and measured results concerning the antenna scattering parameters is reported. From an analysis of this figure it results that while the numerical results show a $38.9 \%$ bandwidth, covering from $1.97 \mathrm{GHz}$ to $2.92 \mathrm{GHz}$, those measured show that the antenna covers a bandwidth of $36.7 \%$, operating from $1.96 \mathrm{GHz}$ to $2.84 \mathrm{GHz}$. In addition, the isolation between the antenna ports, that in the numerical results, is higher than $32 \mathrm{~dB}$ across the entire frequency band and keeps higher than $29 \mathrm{~dB}$ in the measurement results. The slight discrepancies observed between the computed and measured results are mainly due to the fabrication tolerance and the soldering of the SMA connectors employed to excite the antenna.

Since the antenna is symmetrical the radiation patterns related with Port 1 and Port 2 are similar. So, for the sake of brevity only the radiation patterns for Port 1 at 2, 2.4, and $2.8 \mathrm{GHz}$ are illustrated in Figure 13. From these diagrams it can be observed that the main beams are always in the broadside direction without unwanted tilting or splitting. In addition, the measured cross-polarization levels in both $x z$ plane and $y z$-plane are of about $30 \mathrm{~dB}$ lower with respect to the copolarization levels across the entire operating frequency band, while the front-to-back ratios maintain in the range of $25 \pm 3 \mathrm{~dB}$. Finally, the gain and the radiation efficiency presented in Figure 14 show that the antenna achieves a stable gain higher than $8 \mathrm{dBi}$ with an average radiation efficiency of $84 \%$.

The comparisons of the measured performances of the proposed antenna and of those having dual-polarization 


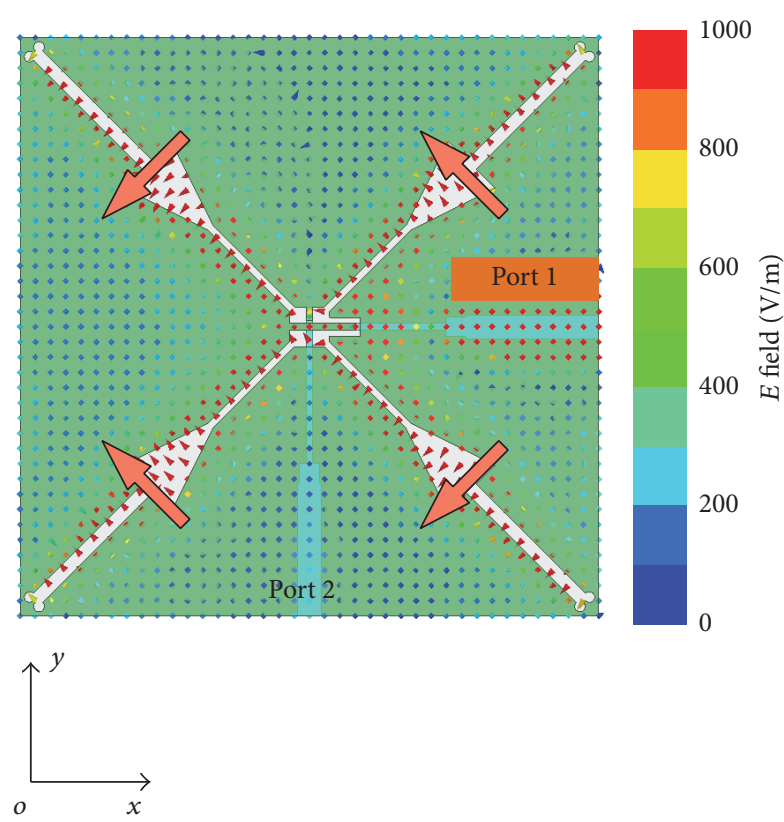

(a)
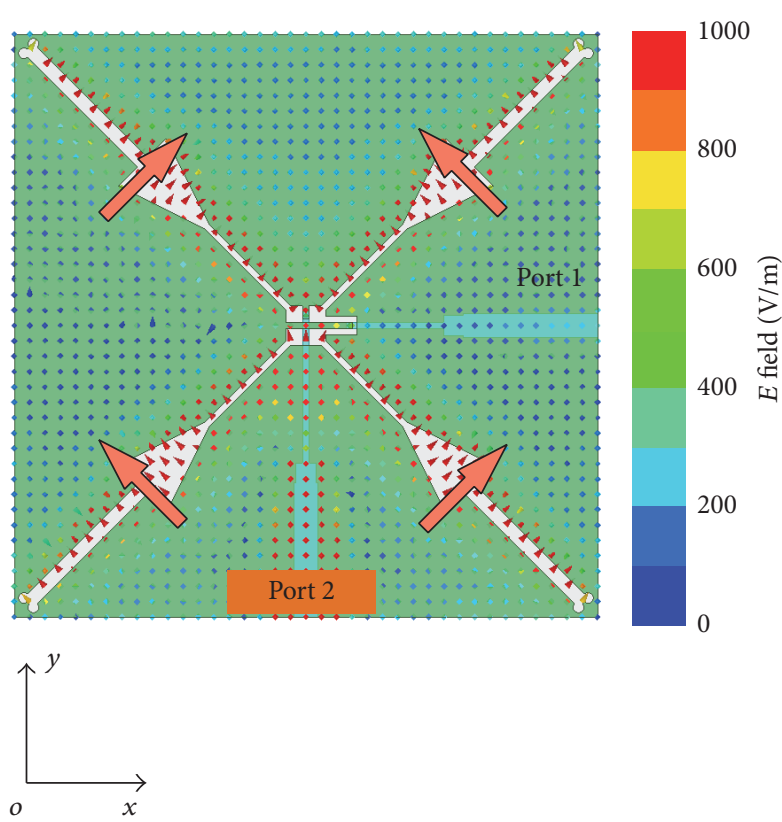

FIgURE 10: Electric-field distributions of the proposed antenna for (a) Port 1 and (b) Port 2.

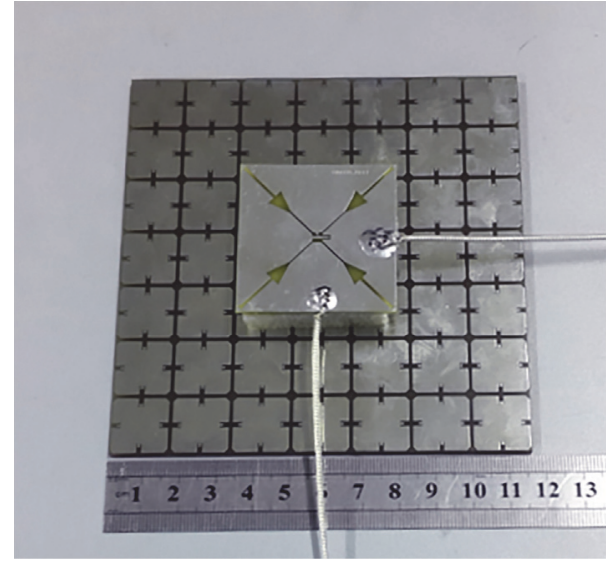

FIGURE 11: Photograph of the proposed dual-polarized slot antenna.

characteristics reported in literature $[1-4,8,11,13,17]$ are listed in Table 2. As it can be observed, the proposed antenna exhibits a wider impedance bandwidth $\left(\left|S_{11}\right|<-10 \mathrm{~dB}\right.$ or VSWR $<2$ ) than other referenced designs listed in Table 2, except for the designs in $[8,11]$. Notably, the antenna profile of our design is only $0.13 \lambda_{c}$, which is lower than that of designs reported in $[8,11]$. Besides, the proposed antenna exhibits stable gain $(>8 \mathrm{~dB})$ and desirable electric isolation ( $>29 \mathrm{~dB}$ ), whose results are good enough for many wireless communication applications.

\section{Conclusion}

A wideband dual-polarized slot antenna excited by two stepped microstrip feedlines has been presented. An AMC surface, acting as reflector, has been employed to drastically



FIGURE 12: Measured and simulated $S$-parameters of the proposed antenna.

reduce the profile of the proposed antenna. The realized prototype exhibits good performances in terms of wide operating band, high electrical isolation, low back side lobes, and stable antenna gain, meaning that it appears a good candidate to be employed in radio base stations for WLAN/WIMAX and LTE applications.

\section{Competing Interests}

The authors declare that there is no conflict of interests regarding the publication of this paper. 

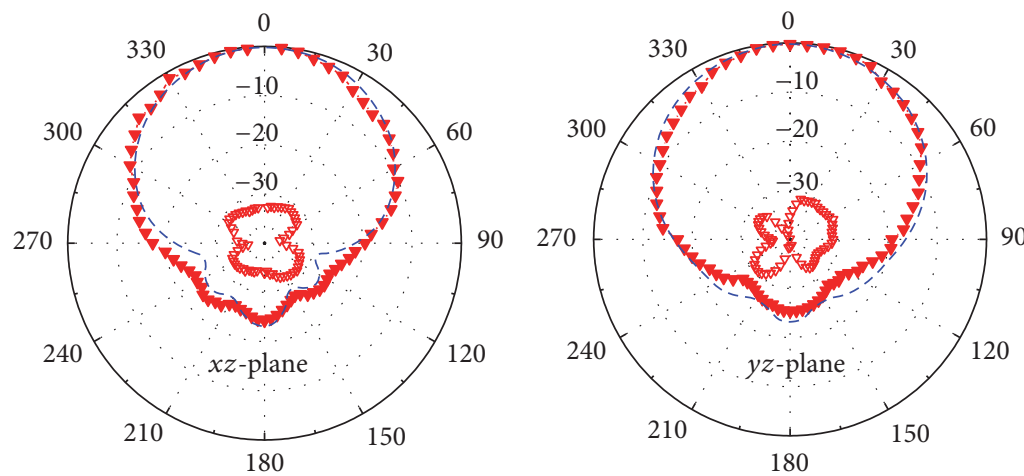

$$
\begin{aligned}
& \nabla \text { Meas. copolar } \\
& -\nabla-\text { Meas. X-polar }
\end{aligned}
$$

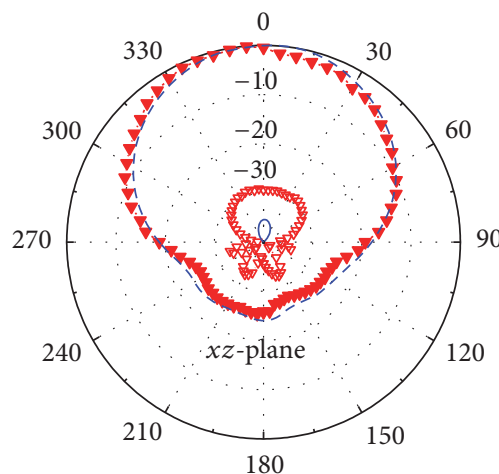

$$
\begin{aligned}
& \neg-\text { Meas. copolar } \\
& -\nabla \text { Meas. X-polar }
\end{aligned}
$$

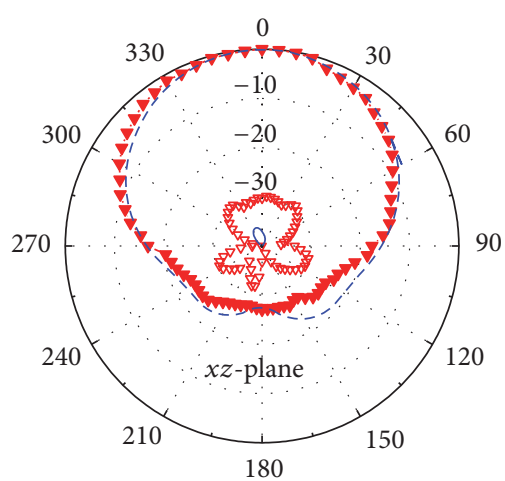

$\nabla$ Meas. copolar
$\nabla-$ Meas. X-polar



- - - Sim. copolar

_ Sim. X-polar

(b)

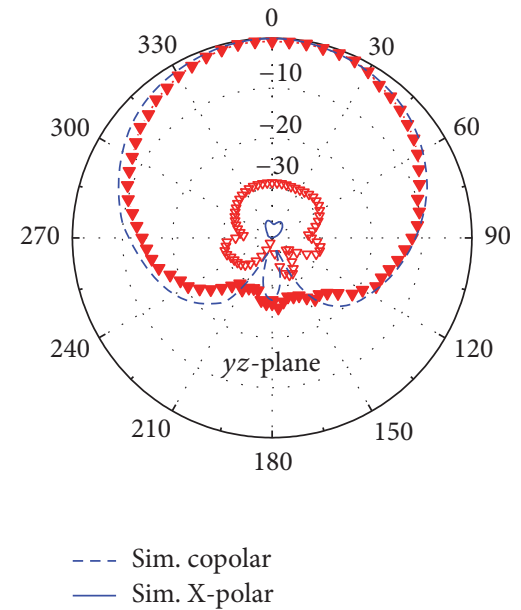

(c)

Figure 13: Radiation patterns for Port 1 of the proposed antenna (a) $2 \mathrm{GHz}$, (b) $2.4 \mathrm{GHz}$, and (c) $2.8 \mathrm{GHz}$. 




FIGURE 14: Measured gain and radiation efficiency of the proposed antenna.

TABLE 2: Comparisons of various dual-polarized antennas.

\begin{tabular}{lcccc}
\hline Ref. & BW, \% & Height, $\lambda_{c}$ & Isolation, dB & Gain, dBi \\
\hline$[1]$ & 14.5 & 0.09 & $>40$ & Around 7 \\
{$[2]$} & 21.3 & 0.11 & $>34$ & $/$ \\
{$[3]$} & 34.9 & 0.19 & $>40$ & $8.7-9.1$ \\
{$[4]$} & 26.0 & 0.11 & $>25$ & Around 10 \\
{$[8]$} & 47.0 & 0.18 & $>37$ & $>8$ \\
{$[11]$} & 67.6 & 0.23 & $>36$ & $6.6-9.6$ \\
{$[13]$} & 28.9 & 0.15 & $>30$ & Around 7 \\
{$[17]$} & 31.2 & 0.15 & $>30$ & $/$ \\
Pro. & 36.7 & 0.13 & $>29$ & $>8$ \\
\hline
\end{tabular}

$\lambda_{c}$ is the wavelength at the center frequency of the working band.

\section{Acknowledgments}

This work was supported by the National Natural Science Foundation of China (no. 61501340).

\section{References}

[1] T.-W. Chiou and K.-L. Wong, "Broad-band dual-polarized single microstrip patch antenna with high isolation and low cross polarization," IEEE Transactions on Antennas and Propagation, vol. 50, no. 3, pp. 399-401, 2002.

[2] S. Gao and A. Sambell, "Dual-polarized broad-band microstrip antennas fed by proximity coupling," IEEE Transactions on Antennas and Propagation, vol. 53, no. 1, pp. 526-530, 2005.

[3] J.-J. Xie, Y.-Z. Yin, J.-H. Wang, and X.-L. Liu, "Wideband dualpolarised electromagneticfed patch antenna with high isolation and low cross-polarisation," IET Electronics Letters, vol. 49, no. 3, pp. 171-173, 2013.

[4] H.-W. Lai and K.-M. Luk, "Dual polarized patch antenna fed by meandering probes," IEEE Transactions on Antennas and Propagation, vol. 55, no. 9, pp. 2625-2627, 2007.

[5] Z.-Y. Zhang, S. Li, S.-L. Zuo, J.-Y. Zhao, X.-D. Yang, and G. $\mathrm{Fu}$, "Dual-polarized crossed bowtie dipole array for wireless communication applications," International Journal of Antennas and Propagation, vol. 2014, Article ID 349516, 8 pages, 2014.
[6] M. Ciydem, "Isolation enhancement in wideband dual-polarised suspended plate antenna with modified T-type probes," Electronics Letters, vol. 50, no. 5, pp. 338-339, 2014.

[7] Y. Liu, H. Yi, F.-W. Wang, and S.-X. Gong, "A novel miniaturized broadband dual-polarized dipole antenna for base station," IEEE Antennas and Wireless Propagation Letters, vol. 12, pp. 1335-1338, 2013.

[8] K. M. Mak, X. Gao, and H. W. Lai, "Low cost dual polarized base station element for long term evolution," IEEE Transactions on Antennas and Propagation, vol. 62, no. 11, pp. 5861-5865, 2014.

[9] Y.-X. Guo, K.-M. Luk, and K.-F. Lee, "Broadband dual polarization patch element for cellular-phone base stations," IEEE Transactions on Antennas and Propagation, vol. 50, no. 2, pp. 251-253, 2002.

[10] R. Caso, A. A. Serra, M. Rodriguez-Pino, P. Nepa, and G. Manara, "A wideband slot-coupled stacked-patch array for wireless communications," IEEE Antennas and Wireless Propagation Letters, vol. 9, pp. 986-989, 2010.

[11] Q. Xue, S. W. Liao, and J. H. Xu, "A differentially-driven dualpolarized magneto-electric dipole antenna," IEEE Transactions on Antennas and Propagation, vol. 61, no. 1, pp. 425-430, 2013.

[12] Y. Luo and Q.-X. Chu, "Oriental crown-shaped differentially fed dual-polarized multidipole antenna," IEEE Transactions on Antennas and Propagation, vol. 63, no. 11, pp. 4678-4685, 2015.

[13] Y.-X. Guo, K.-W. Khoo, and L. C. Ong, "Wideband dualpolarized patch antenna with broadband baluns," IEEE Transactions on Antennas and Propagation, vol. 55, no. 1, pp. 78-83, 2007.

[14] X. Jiang, Z. Zhang, Y. Li, and Z. Feng, "A wideband dualpolarized slot antenna," IEEE Antennas and Wireless Propagation Letters, vol. 12, pp. 1010-1013, 2013.

[15] D. Sievenpiper, L. Zhang, R. F. J. Broas, N. G. Alexöpolous, and E. Yablonovitch, "High-impedance electromagnetic surfaces with a forbidden frequency band," IEEE Transactions on Microwave Theory and Techniques, vol. 47, no. 11, pp. 2059-2074, 1999.

[16] A. Foroozesh and L. Shafai, "Application of combined electricand magnetic-conductor ground planes for antenna performance enhancement," Canadian Journal of Electrical and Computer Engineering, vol. 33, no. 2, Article ID 4621833, pp. 87-98, 2008.

[17] R. N. Lian, Z. D. Wang, Y. Z. Yin, J. J. Wu, and X. Y. Song, "Design of a low-profile dual-polarized stepped slot antenna array for base station," IEEE Antennas and Wireless Propagation Letters, vol. 15, pp. 362-365, 2016.

[18] D. Caratelli, R. Cicchetti, G. Bit-Babik, and A. Faraone, "Circuit model and near-field behavior of a novel patch antenna for WWLAN applications," Microwave and Optical Technology Letters, vol. 49, no. 1, pp. 97-100, 2007. 


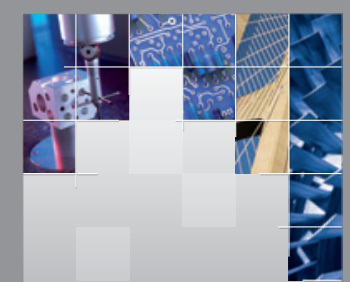

\section{Enfincering}
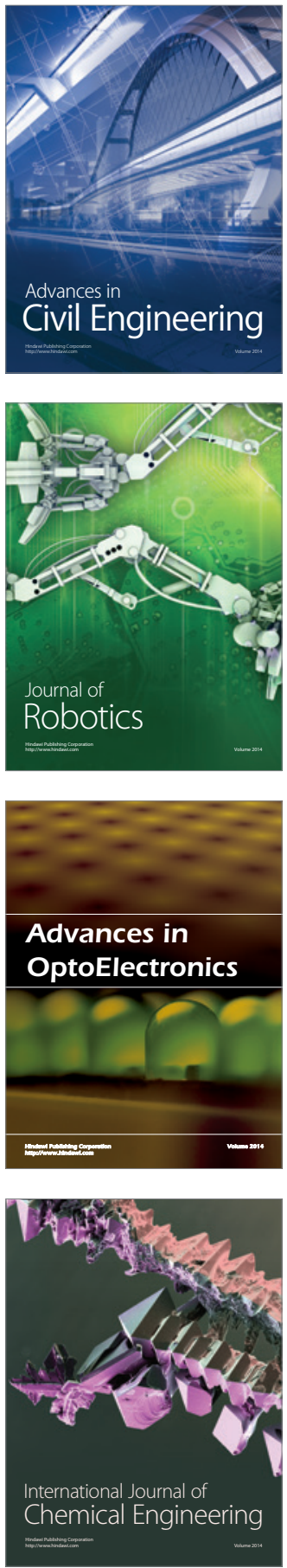

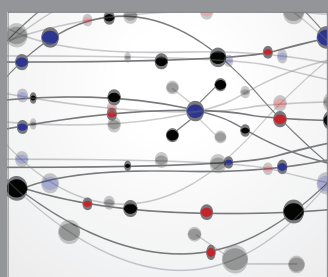

The Scientific World Journal

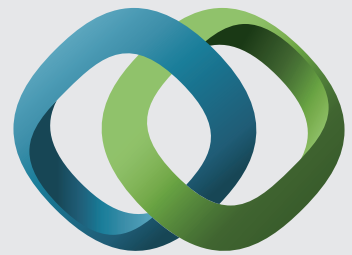

\section{Hindawi}

Submit your manuscripts at

http://www.hindawi.com
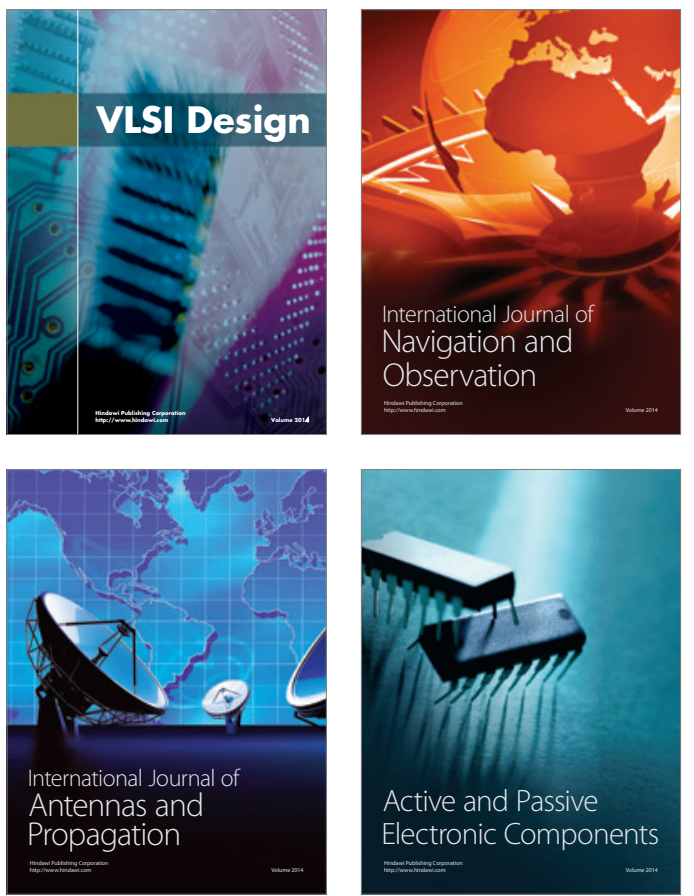


International Journal of

Distributed

Sensor Networks

Journal of

Control Science

and Engineering
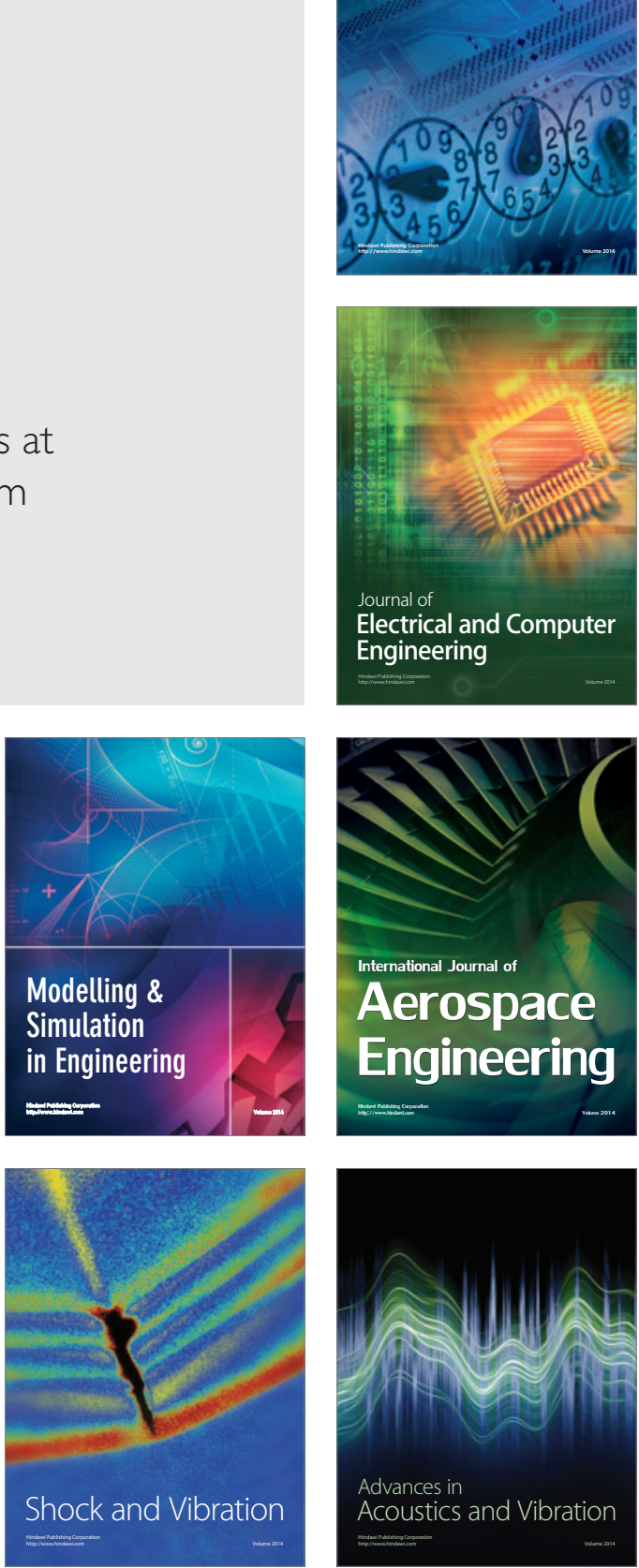